Nerve conduction studies

\begin{tabular}{llllll}
\hline Nerve & $\begin{array}{l}\text { Latency }(\mathrm{ms}) \\
\text { onset }\end{array}$ & $\begin{array}{l}\text { Velocity } \\
(\mathrm{m} / \mathrm{s})\end{array}$ & $\begin{array}{l}\text { Amplitude }(\mathrm{mV}) \\
\text { distal/proximal }\end{array}$ & $\begin{array}{l}\text { Minimum } F- \\
\text { latency }(\mathrm{ms})\end{array}$ & $\begin{array}{l}\text { SNAP amplitude } \\
(\mu \mathrm{V})\end{array}$ \\
\hline Motor: & & & & & \\
R median & $4 \cdot 0$ & $44 \cdot 3$ & $7 \cdot 0 / 6 \cdot 7$ & $30 \cdot 4$ & \\
R ulnar & $4 \cdot 0$ & $54 \cdot 4$ & $6 \cdot 7 / 7 \cdot 1$ & $28 \cdot 9$ & \\
R peroneal & $3 \cdot 9$ & $46 \cdot 1$ & $4 \cdot 1 / 4 \cdot 7$ & $57 \cdot 2$ & \\
R tibial & $3 \cdot 7$ & $43 \cdot 9$ & $11 \cdot 8 / 9 \cdot 5$ & $52 \cdot 7$ & $10 \cdot 7$ \\
Sensory: & $3 \cdot 2$ & $43 \cdot 8$ & & & $25 \cdot 1$ \\
R sural & $3 \cdot 0$ & $46 \cdot 7$ & & & $21 \cdot 0$ \\
R median & $3 \cdot 0$ & $48 \cdot 3$ & & & $20 \cdot 1$ \\
R ulnar & $2 \cdot 9$ & 50.0 & & & \\
R radial & $2 \cdot 0$ & & & \\
\hline
\end{tabular}

SNAP = Sensory nerve action potential; $R=$ right.

Urine output increased to $2000 \mathrm{ml}$ in the next six days. Audiometry on the third day suggested severe sensorineural hearing loss. On day 7 she complained of prickling, tingling, and numbness of the distal limbs, extreme over the feet. The sensory problems became even worse two weeks later.

The patient was fully conscious but had a severe hearing deficit. Her limbs were movable but with generalised hyporeflexia, bilateral plantar flexor responses, impaired sensation to pinprick and light touch at the distal end of the limbs, and intact position and vibration sensations. Nerve conduction studies performed one month later showed decreased nerve conduction velocities and reduced amplitude of compound muscle action potentials and senory action potentials in the limbs (table). No fibrillation potentials or positive sharp waves were found during needle electromyographic examination. Seven months later the distal limbs continued to be numb. Serum urea nitrogen was $26 \mathrm{mg} / \mathrm{dl}$ and serum creatinine was $2 \cdot 2 \mathrm{mg} / \mathrm{dl}$. The patient had recovered from her sensory problems one year after the ingestion. Six months later, muscular strength, reactive tendon reflexes, and sensation in all modalities were normal. She could hear the telephone ringing without aid. The sural nerve conduction studies showed low amplitudes of sensory action potential but normal latencies.

Bromate poisoning has occasionally been described in the medical literature with patients presenting with restlessness, depressed consciousness, and generalised convulsions. It is encountered mainly in children who ingest the agent accidentally and in adults who attempt suicide, and it can produce serious nephrotoxic and ototoxic sequelae..$^{1-3}$ Hearing problems are seemingly less severe in children, probably because of the potential for neural regeneration. Fatal events have mainly been ascribed to acute renal failure.

The estimated lethal dose of potassium bromate ranges from about 200 to 500 $\mathrm{mg} / \mathrm{kg}$ of body weight, equivalent to 10 to $25 \mathrm{~g}$ per person of average body weight. Our patient ingested about $7.5 \mathrm{~g}$ of sodium bromate $(130 \mathrm{mg} / \mathrm{kg}$ body weight), not lethal but toxic enough to cause renal failure and deafness.

She had tinnitus soon after ingestion, and deafness occurred five hours later. Renal failure developed on the second day, and oliguria responded poorly to diuretics. Renal function improved after management by haemodialysis but hearing was permanently damaged. Renal biopsy was not performed, but renal tubular necrosis in the proximal convoluted tubules, interstitial oedema, and inflammation have been reported. ${ }^{1}$ The pathology of the inner ear is not known.

Our patient had prickling and numbness of the limbs, seven days after ingestion. This sensory discomfort lasted for one year, longer than any previous report, and was accompanied by muscular stretch hyporeflexia. Nerve conduction studies confirmed the clinical findings of sensorimotor polyneuropathy.

Permanent cold wave setting solutions contain a thioglycolate hair care lotion and a bromate solution (either $2 \%-4 \%$ potassium bromate or $10 \%-20 \%$ sodium bromate) ${ }^{3}$ The first makes the hair flexible by changing the sulphur-sulphur (S-S) bonds of keratin to sulphur-hydrogen (S-H) bonds. The bromate then reoxidises the S-H bonds to form different S-S bonds and curl the hair. Like chlorate, a strong oxidising agent, bromate can interfere with $\mathrm{S}-\mathrm{H}$ groups in energy generation pathways to result in methaemoglobinaemia, although this may not be manifest. ${ }^{1}$ As suggested by Quick et al, the kidney and cochlea have similar antigenicity. ${ }^{4}$

Spontaneous recovery may occur as a possible result of reformation of S-S bonds. The primary changes may occur in the Schwann cells and the myelin sheaths, as reported in a case with sural nerve biopsy. ${ }^{5}$

Measurement of the serum concentration of bromate is currently not possible. Hence, we measured the bromide concentration of the patient instead of bromate, as neither should be present in normal subjects. High concentrations of bromide may indicate intoxication by bromide; the detectable concentration of bromide in our patient is attributed to conversion from bromate and can be an indicator of residual bromate.

Prognosis after bromate poisoning is usually poor, but our patient recovered from renal failure after haemodialysis. Her sensory complaints had also disappeared one year later, although she remained partially deaf.

VINCHI WANG

Department of Neurology, Cardinal Tien Hospital, Hsintien, Taipei, Taiwan CHING-PIAO TSAIN KO-PIAO TSAI y, Neurological Institute,

Department of Neurology, Neurological Institute,
Veterans General Hospital,
Taipei, Taiwan.

Department of Neurology, Neurological Institute,
Veterans General Hospital,
Taipei, Taiwan. National Yang-Ming Medical College, Taipei,
Taiwan, 11217, Republic of China

Correspondence to: Dr Kon-Ping Lin.

1 Dunsky I. Potassium bromate poisoning. $\operatorname{Am} \mathcal{F}$ Dis Child 1947;74:730-4.

2 Matrumoto I, Morizono T, Paparella MM. Hearing loss following potassium bromate: two case reports. Otolaryngol Head Neck Surg 1980;88:625-9.
3 Lue IN, Johnson CE, Edwards DL. Bromate poisoning from ingestion of professional hair-care neutraliz:

4 Quick CA, Fish A, Brown C. The relationship between cochlea and kidney. Laryngoscope 1973;83:1469-82.

5 Deshimaru M, Miyagawa T, Sumiyoshi $S$, Nomura Y. Peripheral nerve disease associated with acute renal failure due to bromate poisoning. No To Shinkei 1976;28:807-14.

\section{Embolism across the circle of Willis}

Embolism is one of the proposed causes of ischaemic strokes delayed in onset after internal carotid artery (ICA) occlusion. ${ }^{1}$ The embolus may arise from a "tail" of thrombus that lies at the top of the ICA, from thrombus from the "stump" of the ICA, ${ }^{3}$ or from thrombus from the ipsilateral external carotid artery passing through pial vessels of the ophtalmic artery. ${ }^{4}$ Another mechanism could be embolisation across the circle of Willis. To support this hypothesis, transcranial Doppler ultrasonography in four patients with internal carotid artery occlusion detected embolic signals in the middle cerebral artery ipsilateral to the internal carotid artery occlusion. After surgery of the opposite stenosed internal carotid artery no signal was detected .5

We describe a patient with clinical, radiological, and ultrasonographic evidence of left hemispheric infarction by embolic migration through the anterior communicating artery.

A 74 year old right handed man underwent right carotid endarterectomy six years earlier for right transient cerebral ischaemia. Conventional arteriography showed a stenosis greater than $70 \%$ of the right internal carotid artery and an occlusion of the left internal carotid artery. Six years later a right renal artery stenosis was discovered that required transluminal angioplasty. During the procedure, as the axillary catheter was removed through the aorta, the patient sustained a right hemiparesis with aphasia. On admission to the stroke unit of the Salpêtrière Hospital two hours later, the patient was awake, but on neurological examination there was no comprehension of any order or oral expression. He had a right hemiparesis with Babinski's sign, right hemianopsia, and hypoaesthesia. General physical examination, a chest radiograph, routine laboratory investigations, and ECG were normal. Brain CT showed two recent areas of hypodensity taking the contrast in the right frontal and left frontoparietal regions to correspond respectively to pial vessel infarcts in the territory of the right and left MCA (figure). Colour Doppler ultrasound of the neck vessels confirmed the old occlusion of the left internal carotid artery and showed a stenosis of $80 \%$ of the right internal carotid artery with evidence of fresh thrombotic material floating in the lumen. Periorbital directional Doppler detected no reverse flow in the ophtalmic artery. Transcranial Doppler ultrasonography showed full irrigation of the left middle cerebral artery by the anterior communicating artery with an acceleration of velocity in the right middle cerebral artery.

Several mechanisms could be responsible for the ischaemic stroke in this patient. Firstly, it could be caused by embolic migration through an extracranio-orbital anastomosis. The source of embolic material could be directly the left external carotid 


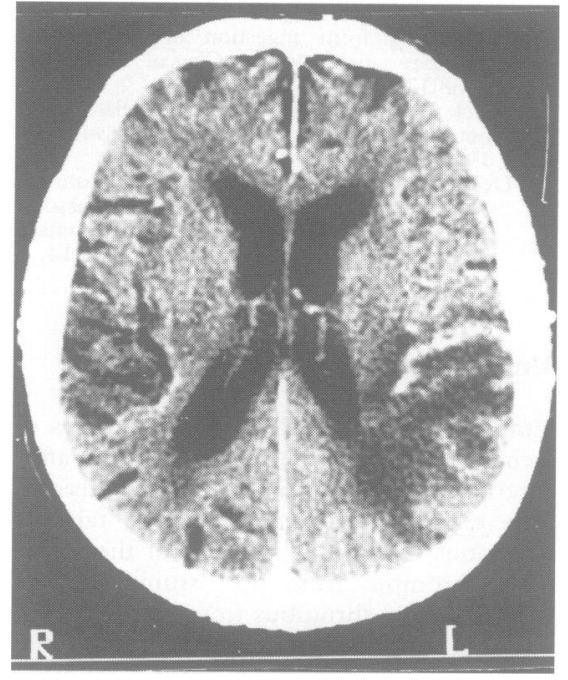

Axial CT with injection of contrast, showing two recent areas of hypodensity in the right frontal and left frontoparietal regions corresponding to pial vessel infarcts in the territory of the right and left middle cerebral artery.

artery, ${ }^{4}$ the proximal carotid "stump" ${ }^{3}$ or, in the iatrogenic circumstances of this patient, the aorta. Periorbital directional Doppler, however, demonstrated normal flow in the ophthalmic artery. Secondly, embolisation through extracraniomeningeal anastomosis could be responsible but it is unlikely as these are considered too narrow to allow an embolus responsible for such a large left hemispheric infarction to pass. A third possibility is embolisation of thrombotic material breaking off from the distal soft "white tail" of the thrombus located in the left internal carotid artery. ${ }^{2}$ This hypothesis is lacking support: there was arteriographic evidence of internal carotid artery occlusion for at least six years and a "soft white tail" has little chance of persisting for six years after occlusion of the internal carotid artery. Fourthly, infarctions might result from haemodynamic alterations in blood flow, but at onset there was no evidence of haemodynamic attacks with a low flow state during the transluminal angioplasty. Furthermore, the two ischaemic areas were not similar to those described in watershed infarcts. ${ }^{6}$ Therefore evidence for cortical low flow infarcts in this patient is lacking.

We believe that the most likely cause of the left hemispheric infarction is an embolism across the circle of Willis, in this case embolisation through the anterior communicating artery caused by thrombotic material broken away from thrombi located either in the aorta or the contralateral, stenosed right internal carotid artery where thrombotic material was floating in the lumen. This hypothesis is strongly supported by the presence of left and right hemispheric infarcts of the same age. Embolism across the circle of Willis seems the only plausible mechanism for left hemispheric infarction in our patient. GÉRALD RANCUREL
Service des Urgences Cérébro-Vasculaires, Hôpital de la Salpêtrière, 47 Bd de l'Hôpital, 75013 Paris, France E KIEFFER

Service de chirugie vasculaire,
Hôpital de la Salpêtrière, Hôpital de la Salpêtrière,

Correspondence to: Dr S Timsit.

1 Coté R, Tayler DW, Barnett HJM. Interna carotid occlusion: a prospective study. Stroke 1983;14:898-902.

2 Finkelstein S, Kleinman GM, Cuneo $R$ Baringer JR. Delayed stroke following Baringer JR. Delayed stroke following
carotid occlusion. Neurology 1980;30:84-8.

3 Barnett HJM, Peerles SJ, Kaufmann JCE. Barnett HJM, Peerles SJ, Kaufmann JCE. of further cerebral ischemia. Stroke 1978, 9:448-56

4 Gertler JP, Cambria RP. The role of the external carotid endarterectomy in the treatmen of ipsilateral internal carotid occlusion: collective review. 7 Vasc Surg 1987;6:158-67.

5 Georgiadis D, Grosset DG, Lees KR Transhemispheric passage of microemboli in patients with unilateral internal carotid artery occlusion. Stroke 1993;24:1664-6.

6 Weiller C, Ringelstein EB, Reiche W, Buell U. Clinical and haemodynamic aspects of lowflow infarcts. Stroke 1991;22:1117-23.

\section{MATTERS ARISING}

Elementary visual hallucinations in migraine and epilepsy

We would like to add a cautionary note to the highly interesting study by Panayiotopoulos $^{1}$ on the different elementary visual hallucinations in migraine and epilepsy. The paper concludes that visual hallucinations in occipital epileptic seizures are predominantly multicoloured as opposed to predominantly black and white patterns in migraine.

To be able to reach this conclusion, there needs to be certainty that the diagnosis was correct. This is most likely the case for the patients with epilepsy as in all there was either evidence of spike and slow wave activity or a structural occipital lobe lesion. The group of patients assigned to the migraine group are, however, not clearly defined. The appreciable difficulty in being able to differentiate between migraine and epilepsy is stated but too little is said about the possibility of false diagnosis in the migraine group. So it is possible that some of the patients diagnosed as having migraine actually have occipital epilepsy. This would in turn falsify the conclusion of the study.

To illustrate the difficulty of ascribing a diagnosis of migraine to patients without evidence of spike and slow wave activity or a structural occipital lobe lesion we refer to a patient we described earlier ${ }^{2}$ who experienced visual hallucinations (distorted vision and false colours). She was repeatedly diagnosed as having migraine. Doppler sonography of the posterior cerebral arteries during symptoms showed increased blood flow velocity typical of local autoregulatory hyperperfusion due to increased neuronal activity. This enabled the diagnosis of migraine to be excluded and a diagnosis of occipital epilepsy to be established. Ictal EEG was non-specifically slowed.

As we do not know how many of the migraine group in Panayiotopoulos's study really had migraine, we urge caution in the interpretation and application of the proposed conclusion.

E WILDER-SMITH Urment of Neurology, University of Bern, Inselspital Bern,
3010 Bern, Switzerland

1 Panayiotopoulos CP. Elementary visual hallucinations in migraine and epilepsy. $\mathcal{F}$ Neuro Neurosurg Psychiatry 1994;58:1371-4

2 Wilder-Smith E, Nirkko A. Contribution of concurrent Doppler and EEG in differentiating occipital epileptic discharges from migraine. Neurology 1991;41:2005-7.

Panayiotopoulos replies:

In my report on elementary visual hallucinations in migraine and epilepsy I thought that I was unduly overemphasising that visual partial epileptic seizures may be misdiagnosed as migraine and the need for a precise description of the visual hallucinations in these two conditions. If anything, I was biased stressing the possibility of falsely diagnosing migraine instead of epilepsy rather than the other way round. Two out of the four illustrative cases were selected to demonstrate this diagnostic error.

Therefore, I thank Wilder-Smith for his letter, which reassured me that my above fears were unfounded as he stresses the same point-namely, that visual partial seizures may be misdiagnosed as migraine. He goes one step further however, arguing that some of my patients diagnosed with migraine may have had occipital epilepsy. I do not think that this mistake was made because in all 50 patients the diagnosis of migraine was based on strict clinical criteria, a long follow up, response to treatment, and not only on a normal or equivocally abnormal EEG. In particular, all 47 patients with classic migraine had the characteristic migrainous visual prodrome lasting 5-20 minutes before the onset of mainly unilateral headache characteristic of migraine. Not a single patient in the migraine group had any suggestion of epileptic seizures, which, given my special interest in these conditions, ${ }^{12}$ I would be able to recognise.

The author also wishes to discuss his published case which, like my cases, was misdiagnosed as migraine. I did not cite his report because although the "coloured" visual hallucinations of this patient were consistent with my findings, misdiagnosis was not indicated and previous attacks were monolectically described as "migraine". More clinical details along the lines of my report and previous reports ${ }^{1}$ from WilderSmith would be more enlightening. The patient had clusters of " $15-30$ second attacks of distorted vision and false colours" associated with simultaneous and equally brief ictal EEG changes. The diagnosis of visual partial seizures should be clear and if these were of acute onset in adult life, MRI instead of Doppler would be more appropriate. More confidence in the clinical symptoms, which is the main point of my report, may have avoided the need for further investigations and delaying treatment. 